\title{
Foliar Application of Several Nitrogen Sources as Fertilisers to Tempranillo Grapevines: Effect on Wine Volatile Composition
}

\author{
P. Rubio-Bretón ${ }^{1,2}$, G. Gutiérrez-Gamboa ${ }^{1,2}$, E.P. Pérez-Álvarez ${ }^{1,2}$, P. Santamaría ${ }^{2}$, T. Garde-Cerdán ${ }^{1,2, *}$ \\ (1) VIENAP Group \\ (2) Instituto de Ciencias de la Vid y del Vino (CSIC, Gobierno de La Rioja, Universidad de La Rioja), Logroño, Spain
}

Submitted for publication: January 2018

Accepted for publication: June 2018

Keywords: Aroma compounds, nitrogen applications, phenylalanine, urea, doses, grape

Foliar nitrogen applications to the vineyard make it possible to improve grape and wine phenolic and amino acid concentration. However, to our knowledge, there is little information about their impacts on the volatile composition of wine. Therefore, the aim of this work was to evaluate the effects of the application of several nitrogen sources in Tempranillo vineyards on wine volatile composition during the first season. Subsequently, two dosages of phenylalanine and urea were applied to the vineyard with the aim of evaluating their effect on wine volatile composition. The results show that the different nitrogen sources applied to the grapevines during the first season did not affect higher alcohols, fatty acids, esters and other volatile compounds in the wines. The two dosages of phenylalanine and urea applied to the vineyard during the second vintage barely affected wine volatile composition. Consequently, this study shows that foliar nitrogen treatments in vineyards did not modify the wine aromatic profile.

\section{INTRODUCTION}

The nitrogen status of the vineyard directly affects grapevine yield and growth responses, which strongly affect grape composition (Soubeyrand et al., 2014). Several factors affect grapevine nitrogen status, including climate and soil conditions, viticultural practices, and nitrogen form, timing and rate of application (Bell \& Henschke, 2005; Verdenal et al., 2015; Hannam et al., 2016; GutiérrezGamboa et al., 2017a), as well as cultivar and vintage (Gutiérrez-Gamboa et al., 2017b). Amino acids and ammonium play a key role in yeast population dynamics, the correct development of alcoholic fermentation, and the formation of volatile compounds, such as higher alcohols, esters and some fatty acids (Vilanova et al., 2007; GardeCerdán \& Ancín-Azpilicueta, 2008). Nitrogen deficiencies in musts prior to alcoholic fermentation can lead to stuck or sluggish fermentations (Bisson \& Butzke, 2000), and the release of undesirable thiols responsible of sulphurous off-flavour formation (Smith et al., 2015). Thus, nitrogen supplementation in the vineyard is carried out in order to guarantee vine development and a correct grape and must concentration of nitrogen.

Nitrogen fertilisation has traditionally been performed by adding fertiliser to the soil, to be absorbed as nitrate by the shallow plant roots (Christensen \& Peacock, 2000). As nitrate and other salts move into the soil solution, nitrogen concentrations vary drastically, depending on the distance and the depth from the nearest drip emitter or furrow, and they are also altered with each irrigation or rain event (Christensen \& Peacock, 2000). Therefore, it is necessary to develop new fertilisation techniques that allow more precise and effective applications. Recently, foliar fertilisation has been implemented as a new fertilisation technique, as it ensures the efficient assimilation of applied products by the plant (Lasa et al., 2012), which reduces the costs and contributes to sustainable, eco-friendly agriculture. Furthermore, some studies on foliar treatments applied to grapevines have shown differences in grape and wine composition (Hannam et al., 2016; Gutiérrez-Gamboa et al., 2017c). However, there have only been a few reports on the effect of nitrogenous foliar fertilisation on the volatile composition of wine (AncínAzpilicueta et al., 2013; Gutiérrez-Gamboa et al., 2018).

Thus, it was considered of importance to study the effect on wine volatile composition of the foliar application to the vineyard of several nitrogen sources. In relation to the nitrogen sources applied to the grapevines, it was observed that phenylalanine and urea treatments provided the best results. When applied to the vineyard, these two nitrogen compounds improved the concentration of several amino acids in the grapes (Garde-Cerdán et al., 2014a). Furthermore, phenylalanine enhanced the varietal aroma composition of grapes (Garde-Cerdán et al., 2015a). In addition, foliar urea and phenylalanine applications improved the stilbene content in grapes and wines (Garde-Cerdán et al., 2015b).

Due to the aforementioned, the aim was to evaluate

*Corresponding author: E-mail address: Teresa.GardeCerdan@gmail.com; teresa.garde@icvv.es

Acknowledgments: Many thanks for the financial support given by the Gobierno de La Rioja to projects R-11-12 and R-08-13. P. R.-B., E.P. P.-A. and T. G.-C. thank MINECO for the contracts. G. G.-G. thanks CONICYT for the financial support given through, BCH/Doctorado-72170532 
the effect of several nitrogen sources, such as proline, phenylalanine, urea and two different commercial nitrogen products, applied to Tempranillo grapevines on wine volatile compounds during the first season. In addition, the effect of applying phenylalanine and urea, at two dosages to the grapevines, on wine volatile composition was investigated during the second season.

\section{MATERIALS AND METHODS}

\section{Samples, commercial nitrogen fertilisers, grapevine} treatments and vinification

Red grapes from the Vitis vinifera L. Tempranillo variety grown in the experimental vineyard of the Spanish northern region of La Rioja during the 2012 and 2013 vintages were used. In the first season, a control (untreated) and five treatments were carried out using several nitrogen sources: proline (Pro), phenylalanine (Phe), urea (Ur), and two commercial nitrogen fertilisers, without $(\mathrm{Cp})$ and with amino acids (Cpaas) in their composition, according to Garde-Cerdán et al. (2014a). Briefly, the treatments were applied to the grapevine at véraison and one week later. For each application, $200 \mathrm{ml} /$ plant was sprayed over the leaves, which amounted to $0.9 \mathrm{~kg}$ total $\mathrm{N} / \mathrm{ha}$. Four treatments were carried out using Phe and Ur at two different doses: $0.9 \mathrm{~kg}$ N/ha (Phe1 and Ur1), and $1.5 \mathrm{~kg} \mathrm{~N} / \mathrm{ha}$ (Phe2 and Ur2) during the 2013 season. In both years, the treatments were applied in triplicate and were arranged in a completely randomised block design with three vines per replicate.

The grapes were harvested at optimum maturity, followed by destemming and crushing. Subsequently, $3 \mathrm{~kg}$ of pomace (must, seed and skin) were introduced into $4 \mathrm{~L}$ glass bottles (Sampaio et al., 2007). Potassium metabisulfite was added to the samples to give a final total $\mathrm{SO}_{2}$ concentration of $50 \mathrm{mg} / \mathrm{L}$, and then the alcoholic fermentation was initiated by inoculating the pomace with the commercial Saccharomyces cerevisiae strain Uvaferm VRB (Lallemand, St. Simon, France) at a dosage of $20 \mathrm{~g} / \mathrm{hl}$. The fermentations were performed at a controlled temperature of $22^{\circ} \mathrm{C}$. The end of alcoholic fermentation was determined by measuring the reducing sugars using an automatic enzymatic equipment Miura One (Tecnología Difusión Ibérica, Barcelona, Spain). At a later stage, aliquots of each wine were frozen in order to determine their volatile composition.

\section{Analysis of volatile compounds by gas chromatography}

The analysis of the fermentative volatile compounds of the wines was performed using the method described by GardeCerdán et al. (2014b). Firstly, an extraction was carried out by mixing $3 \mathrm{ml}$ of sample, $9.5 \mathrm{ml}$ of $\left(\mathrm{NH}_{4}\right)_{2} \mathrm{SO}_{4}$ saturated solution, $15 \mu \mathrm{l}$ of internal standard solution (2-butanol, 4-methyl-2-pentanol, 4-hydroxy-4-methyl-2-pentanone, 2-octanol and heptanoic acid; $40 \mathrm{mg}$ of each compound/100 $\mathrm{ml}$ of ethanol) and $200 \mathrm{ml}$ of dichloromethane in $15 \mathrm{ml} \mathrm{screw-}$ cap centrifuge tubes. Thereafter, the tubes were shaken for 1 hour at $400 \mathrm{rpm}$ and then centrifuged at $2500 \mathrm{rpm}$ for 10 min. Once the phases were separated, the dichloromethane phase was recovered with a $0.5 \mathrm{ml}$ syringe and transferred to a $0.3 \mathrm{ml}$ vial. Two $\mu \mathrm{l}$ of the extract were injected into a Hewlett-Packard (Palo Alto, CA, USA) 6890 series II gas chromatograph equipped with an automatic injector (Agilent
6890 Series Injector) and a Hewlett-Packard flame ionisation detector (FID). Separation was carried out with a DB-Wax capillary column $(60 \mathrm{~m}$ x $0.32 \mathrm{~mm}$ I.D., x $0.5 \mathrm{~mm}$ film thickness; J\&W Scientific, Folsom, CA, USA). The injection was in split/splitless mode without division for $30 \mathrm{~s}$. The temperature program was as follows: $40^{\circ} \mathrm{C}$ for $5 \mathrm{~min}$, then increased to $220^{\circ} \mathrm{C}$ at a rate of $3^{\circ} \mathrm{C} / \mathrm{min}$. The carrier gas was $\mathrm{N}_{2}$ at a flow rate of $3 \mathrm{ml} / \mathrm{min}$. Split flow was $30 \mathrm{ml} / \mathrm{min}$, injector temperature was $220^{\circ} \mathrm{C}$ and detector temperature was $280^{\circ} \mathrm{C}$. The identification of compounds was carried out by comparison of their retention times with those of pure reference standards using a Hewlett-Packard GCD Series II Gas Chromatograph Electron Ionisation Detector with the same chromatographic conditions. The quantification of the volatile compounds was performed using an internal standard method. Odour activity value (OAV) was calculated as the ratio between the concentration of the individual compound and the perception threshold found in the literature (Vilanova et al., 2009, 2013).

\section{Statistical analysis}

The statistical elaboration of the data was performed using the SPSS Version 21.0 statistical package for Windows (SPSS, Chicago, USA). The treatments were performed in triplicate, so the results for volatile compounds correspond to the mean of three analyses $(n=3)$. The data on the volatile compounds were processed using the analysis of variance (ANOVA). Differences between means were compared using the Duncan test at the 0.05 probability level. Principal component analysis (PCA) was performed on volatile compounds in the different wines using InfoStat Professional 2012 version (InfoStat, www.infostat.com.ar).

\section{RESULTS AND DISCUSSION}

\section{Effect of applications of several nitrogen sources on wine} volatile composition in the first study season

It is known that volatile compounds with OAVs higher than 1 indicate a possible contribution to the wine aroma (Vilanova et al., 2009). Moreover, compounds with OAVs lower than $1(0.2<\mathrm{OAV}<1.0)$ could also contribute to the aroma of wines (Garde-Cerdán et al., 2008). During the 2012 season, the most odoriferous compounds in the Tempranillo wines were ethyl octanoate, isoamyl acetate, acetaldehyde, ethyl hexanoate and ethyl butyrate, while the most abundant compounds were isoamyl alcohols, n-propanol, isobutanol, ethyl acetate and 2-phenylethanol (Tables 1 and 2). The least odorant compounds were diethyl succinate, benzyl alcohol, $n$-butanol, acetoine and hexyl acetate, while the least abundant compounds were ethyl propionate, diethyl succinate, hexyl acetate, 2-phenylethyl acetate and ethyl isobutyrate (Tables 1 and 2). The aromatic description of each compound is presented in Tables 3 and 4. Ferreira et al. (2000) showed that Tempranillo wines presented a high content of eugenol, guaiacol, isoamyl acetate and 2-phenylethyl acetate, and minimum concentrations of 2,3-butanodione, linalool, isovaleric acid, isobutanol, isoamyl alcohol, 2-phenylethanol and methionol.

The higher alcohol and fatty acid contents of wines were hardly affected by the different nitrogen sources applied foliarly to the grapevines (Table 1). Thus, the content in 


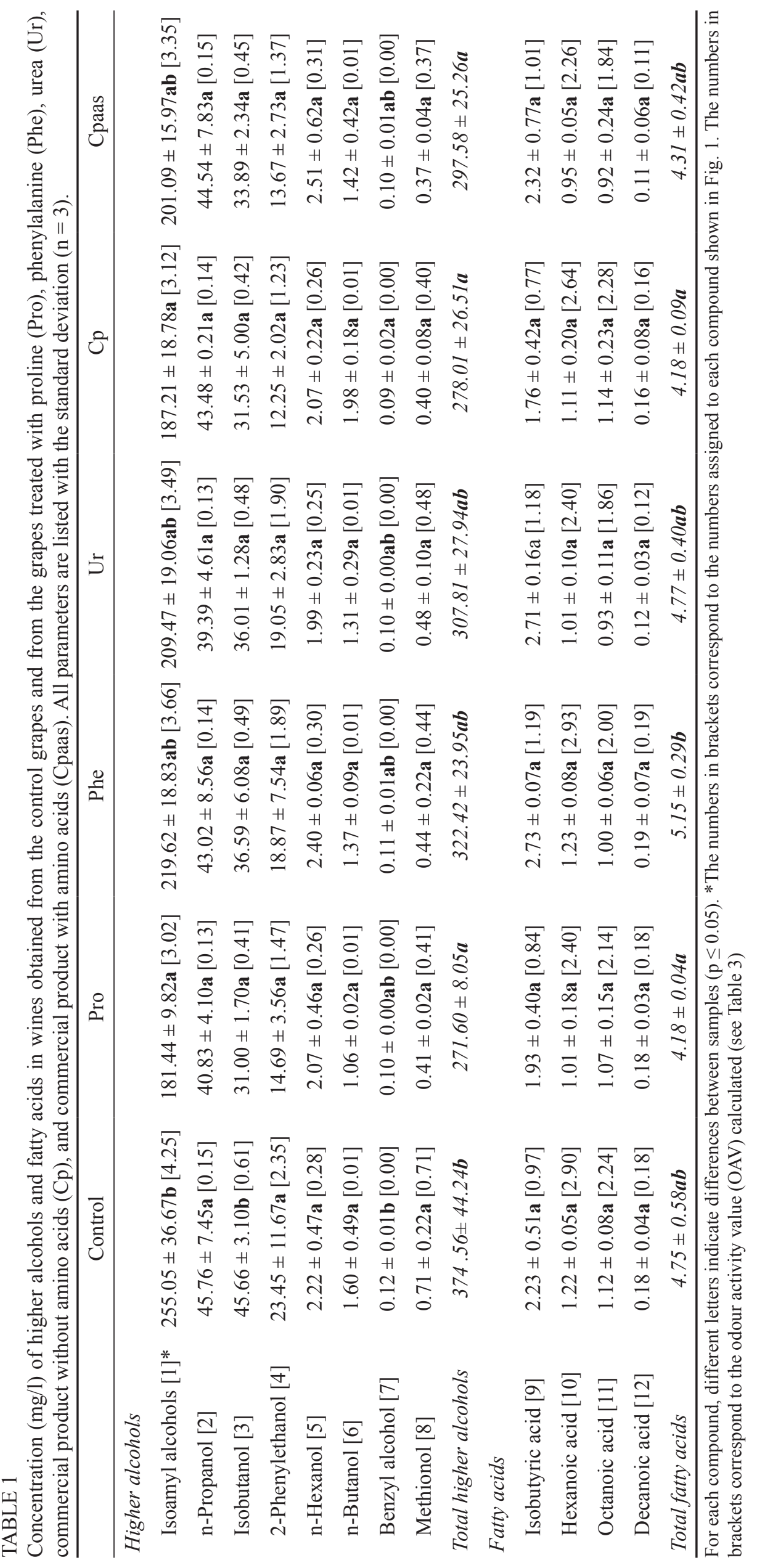




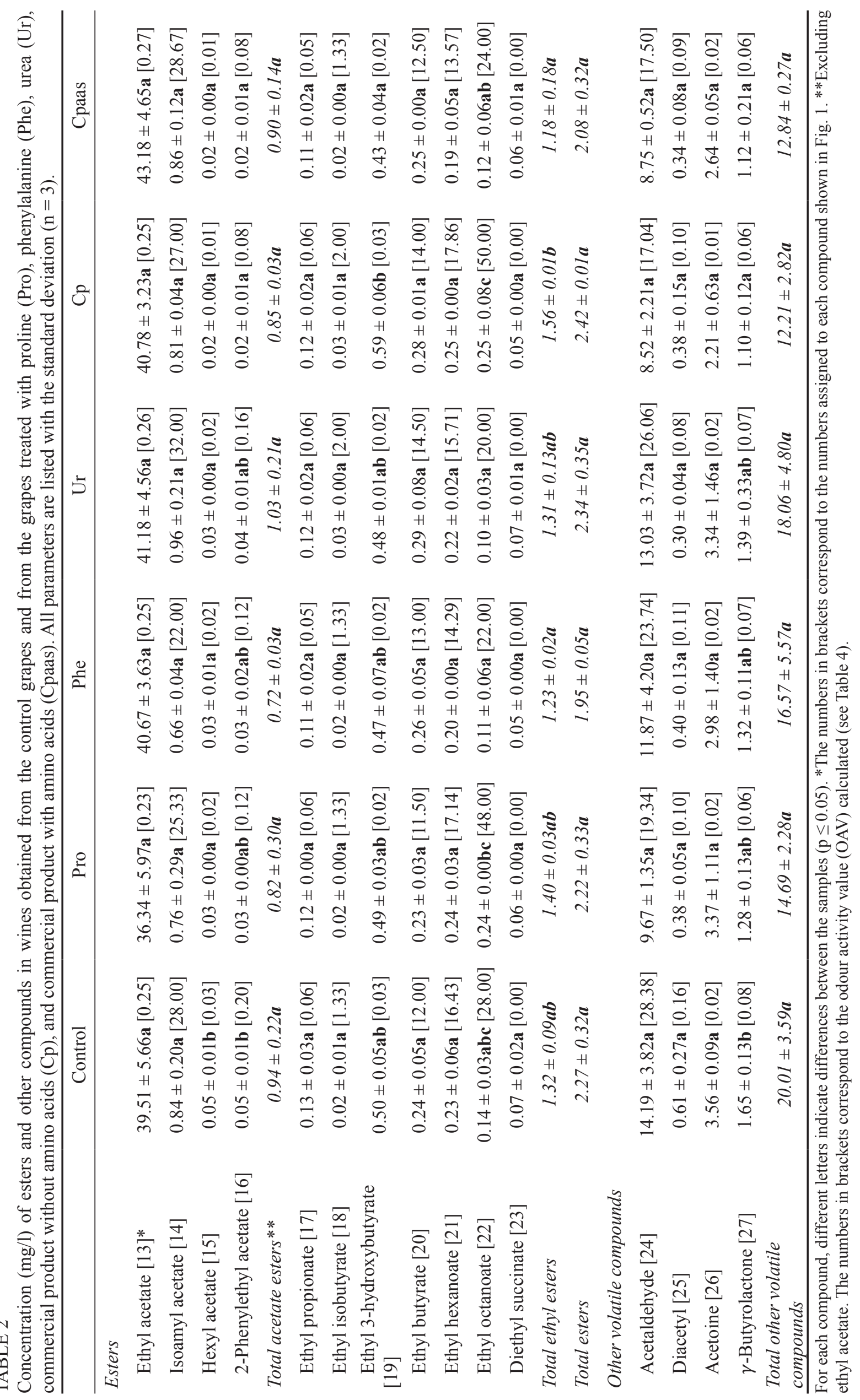


wines of n-propanol, 2-phenylethanol, n-hexanol, n-butanol, methionol, and isobutyric, hexanoic, octanoic and decanoic acids was not affected by the treatments carried out in the vineyard. The wines made from untreated grapes presented higher concentrations of isoamyl alcohols (3-methyl-1butanol + 2-methyl-1-butanol) and benzyl alcohol than the wines from grapevines treated with Pro and $\mathrm{Cp}$, with the exception of benzyl alcohol for the Pro treatment. Besides, the isobutanol content in wines from untreated grapes was the highest. The total content of higher alcohols in wines from untreated grapes was higher than the wines from grapes treated with Pro, Cp and Cpaas. Many of the fatty acids in wines were affected by the treatments carried out in the vineyard. However, the total fatty acid concentration was higher in wines produced from grapes treated with Phe than those treated with Pro and $\mathrm{Cp}$.

With respect to the effects of the different nitrogen sources applied to the grapevines on ester and other volatile compound in the wines, it seems that the applications barely affected their content (Table 2). The concentration of ethyl acetate, 2-phenylethyl acetate, ethyl propionate, ethyl isobutyrate, ethyl butyrate, ethyl hexanoate, diethyl succinate, acetaldehyde, diacetyl and acetoine was not affected by the treatments applied to the vineyard. The control wines presented the highest hexyl acetate content. The wines from grapevines treated with commercial products (Cp and Cpaas) showed lower 2-phenylethyl acetate and $\gamma$-butyrolactone contents than the wines from the untreated grapevines. The total acetate esters content in the wines was not affected by the treatments conducted in the vineyard. The wines from grapes treated with $\mathrm{Cp}$ presented a higher ethyl 3-hydroxybutyrate content than Cpaas, and a higher ethyl octanoate concentration than the wines elaborated from grapes treated with Phe, Ur and Cpaas. Total ethyl esters in wines from grapes treated with $\mathrm{Cp}$ were higher than in the Phe and Cpaas wines. However, the content of total esters and total other volatiles was not affected by the treatments carried out in the vineyard.

Several nitrogen sources have been applied to the vineyard with the aim of improving grape quality; however, there is little information about their effects on the volatile composition of grapes and especially of wine. Lacroux et al. (2008) reported that foliar applications of urea, and mainly urea plus sulphur treatment of Sauvignon blanc grapevines, allowed a higher thiol concentration in wines than the samples from untreated grapevines. Gutiérrez-Gamboa et al. (2018) reported that the wines from grapevines treated with arginine presented a lower total content of higher alcohols and acetate esters than wines from untreated grapevines. In addition, urea and urea plus sulphur treatments applied to the grapevines gave rise to a lower total ethyl ester content in the wines compared to the control samples. In this work, the total terpene content of the wines improved after urea and arginine applications to the grapevines.

Nitrogen supplementation to the grapes influences the production of volatile compounds, mainly by yeast and other microorganisms (Swiegers et al., 2005). Nitrogen deficiencies in musts lead to low yeast populations and poor fermentation vigour, which results in risks of sluggish, stuck or slow alcoholic fermentations (Bisson \& Butzke, 2000).
This problem leads to an increase in the release of undesirable thiols and higher alcohols, with a low production of esters and long-chain volatile fatty acids (Carrau et al., 2008). Ethyl and acetate esters have complex associations with nitrogen availability due to their different synthetic origins (Vilanova et al., 2007). However, ethyl acetate content in wines has been positively related to the must nitrogen concentration (Ancín-Azpilicueta et al., 2013; GutiérrezGamboa et al., 2018). On the other hand, it has been shown that branched-chain fatty acids and their esters are associated with low nitrogen concentrations, whereas medium-chain fatty esters and acetic acid are associated with high nitrogen concentrations (Vilanova et al., 2007). Despite the fact that the foliar application of phenylalanine and urea in the first study season improved the concentration of several amino acids, such as serine, threonine, tyrosine, methionine, tryptophan, phenylalanine, isoleucine, leucine, alanine, arginine, total amino acids and total amino acids without proline, as was showed by Garde-Cerdán et al. (2014a), the concentration of most of the volatile compounds, including higher alcohols and ethyl esters, presented in this manuscript was scarcely affected by the different applications. Based on this, Ugliano et al. (2009) reported that, for the Sacharomyces cerevisiae yeast strain, the addition of diammonium phosphate (DAP) to a final yeast assimilable nitrogen (YAN) of 250 or $400 \mathrm{mg} / \mathrm{L}$ resulted in an increase in sulphides, bisulfides, mercaptans and mercaptoesters compared to non-supplemented fermentations (100 mg/L YAN). Moreover, Barbosa et al. (2009) showed that the addition of $200 \mathrm{mg} \mathrm{N} / \mathrm{L}$ to a must with an initial YAN of $67 \mathrm{mg} \mathrm{N} / \mathrm{L}$ at stationary phase allowed to increases the content of 2-phenylethanol, ethyl isobutyrate, 2-phenylethyl acetate, ethyl 2-methylbutyrate, ethyl propionate, isovaleric acid, isoamyl alcohol and ethyl isovalerate in wines, depending on the strain used. According to the showed by Garde-Cerdán et al. (2014a) in this samples, YAN ranged from 169.4 to $282.8 \mathrm{mg} \mathrm{N} / \mathrm{L}$. Therefore, the musts contained enough nitrogen to carry out a correct alcoholic fermentation, and it is probable that the small differences in nitrogen composition of the samples did not allow statistical differences in wine volatile composition, which were mainly in the esters and higher alcohols and were produced by the sugar and nitrogen metabolism of yeast activity.

\section{Effect of two doses of phenylalanine (Phe) and urea (Ur) applications on wine volatile composition in the second study season}

Due to the positive effects of the Phe and Ur treatments (Garde-Cerdán et al., 2014a, 2015a, 2015b), four treatments were carried out using Phe and Ur at two different dosages: $0.9 \mathrm{~kg} \mathrm{~N} / \mathrm{ha}$ (Phe1 and Ur1), and $1.5 \mathrm{~kg} \mathrm{~N} / \mathrm{ha}$ (Phe2 and Ur2), in order to study the effect of these dosages on the volatile composition of the wine. During the 2013 season, the compounds in Tempranillo wines with the most smell were acetaldehyde, isoamyl acetate, ethyl octanoate, ethyl hexanoate and ethyl butyrate, while the most abundant compounds were isoamyl alcohols, ethyl acetate, acetoine, n-propanol and isobutanol (Tables 3 and 4). The least odorant compounds were diethyl succinate, benzyl alcohol, n-butanol, acetoine and hexyl acetate, while the least 


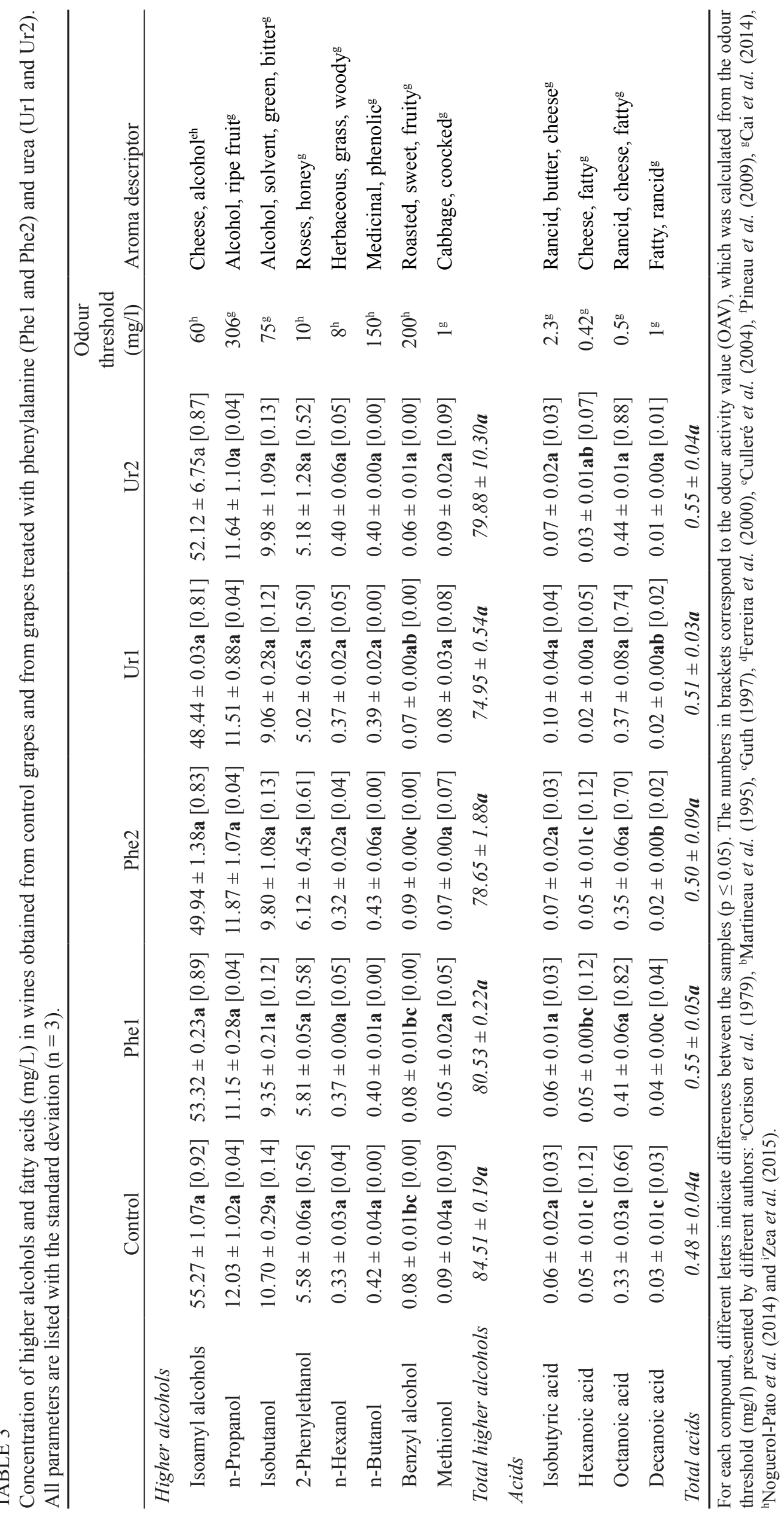




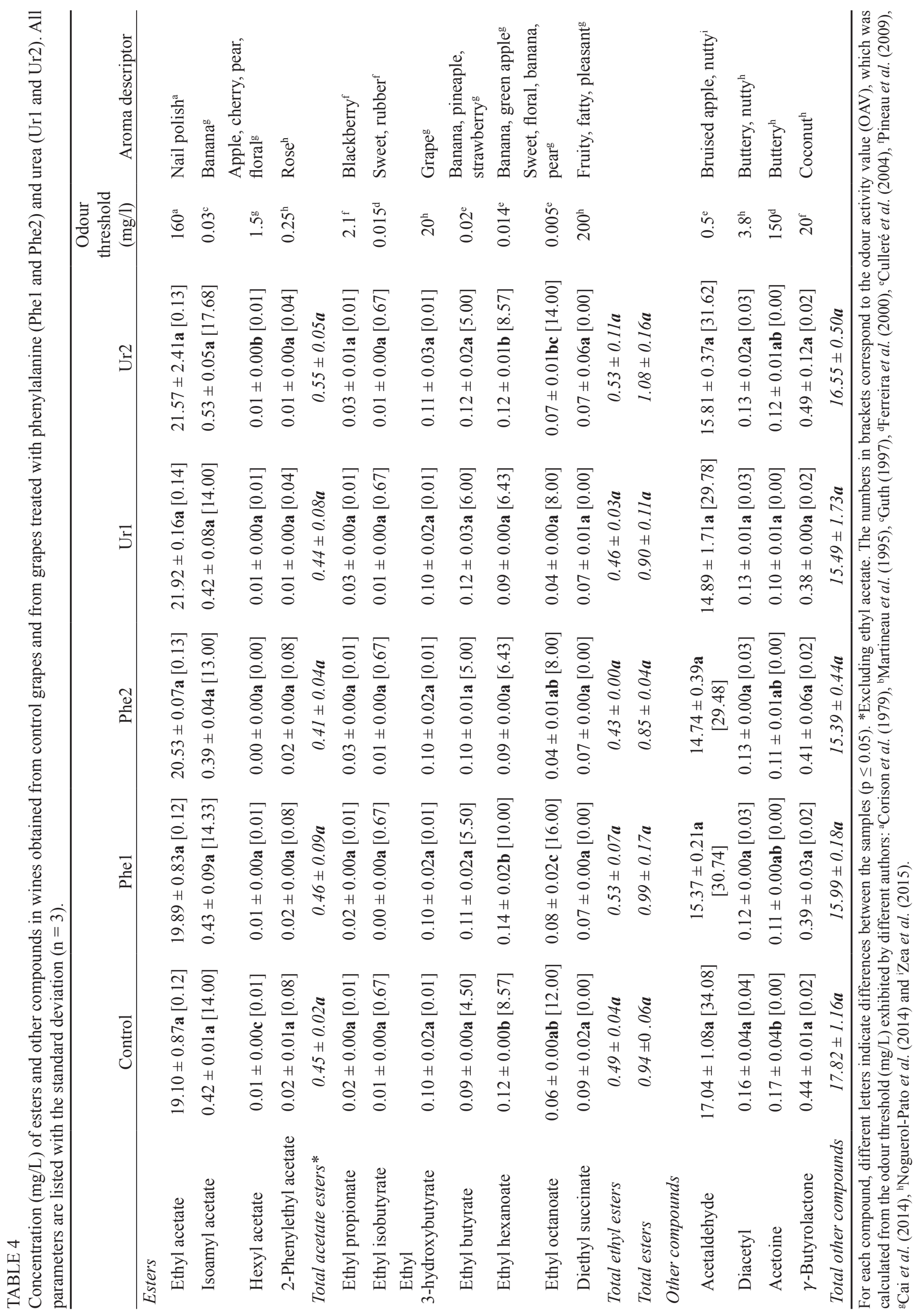


abundant compounds were decanoic acid, 2-phenylethyl acetate, ethyl propionate, hexyl acetate and ethyl isobutyrate (Tables 3 and 4). Based on this, the most important aromatic attributes in Tempranillo wines were mainly fruit descriptors (OAVs higher than 1). Noguerol-Pato et al. (2014) showed that ripe fruit and fresh fruit were the most important aromatic attributes in all red wines, including Tempranillo, followed by lactic, floral, vinous, spicy and herbaceous nuances.

The concentration of higher alcohols and fatty acids in wines was hardly affected by the treatments applied to the grapevines foliarly (Table 3). The content of all the higher alcohols in wines, except benzyl alcohol, was not affected by the treatments applied. The concentration of benzyl alcohol in wines from grapes treated with the high dose of Phe (Phe2) was higher in respect to the wines from grapes treated with both doses of urea. The total content of higher alcohols was not affected by the treatments applied to the vineyard. These results are in agreement with those reported by GutiérrezGamboa et al. (2018), showing that the use of only a foliar application of Arg decreased total higher alcohols, while other nitrogen sources applied to the vineyard, such as urea, urea plus sulphur and commercial nitrogen products, did not affect higher alcohols in the wines. Wines from grapes treated with Ur1 showed a lower hexanoic acid content than wines made from grapes untreated and treated with both doses of Phe (Phe1 and Phe2), while wines from the latter treatment showed a higher decanoic acid concentration than wines from grapes treated with Ur2. Total fatty acids content was not affected by the treatments applied to the vineyard. In this way, Ancín-Azpilicueta et al. (2013) showed that, in general, a low dosage of urea decreased fatty acid content in wines, and the highest dosage of urea applied to the grapevines did not affect total fatty acid compared to the control wines.

Both doses had a noticeable effect on esters and other volatile compounds in the wines (Table 4). The concentration of ethyl acetate, isoamyl acetate, 2-phenylethyl acetate, ethyl propionate, ethyl isobutyrate, ethyl 3-hydroxybutyrate, ethyl butyrate, diethyl succinate, acetaldehyde, diacetyl, $\gamma$-butyrolactone, total acetate esters, total ethyl esters, total esters and total other volatile compounds was not affected by the treatments conducted in the vineyard. The hexyl acetate content in the wines from untreated grapes was the highest. However, its concentration in wines from grapes treated with Ur2 was higher than in wines from grapes treated with both doses of Phe and Ur1. C6 compounds such as hexan1-ol, hexenal, (E)-2-hexen-1-ol and (E)-2-hexenal are all precursors of hexyl acetate (Dennis et al., 2012). According to the findings of Garde-Cerdán et al. (2015a), grapes from untreated grapevines have a higher content of hexanal, (E)-2hexenal and total C6 compounds than grapes from grapevines treated with Phe, and non-statistical differences were found compared to the urea applications in the first study season. The ethyl hexanoate content in wines from grapes treated with Phe 2 and Ur1 was lower than in the wines from grapes treated with the rest of the applications. The wines from grapes treated with Phel showed higher ethyl octanoate content than the control, Phe 2 and Ur1 samples. In addition, wines from untreated grapes had a higher acetoin content than wines from grapes treated with the lowest dose of urea. Acetoin is formed during fermentation by the microbial activity of lactic bacteria and yeast. Sacharomyces strains produce a-acetolactate, which is converted to acetoin during fermentation in the presence of oxygen. The formation of a-acetolactate is related to the consumption of carbohydrates, threonine and lysine, and the potassium and iron cations present in the medium (Romano \& Suzzi, 1996). However, according to the research by Garde-Cerdán et al. (2014a), the lowest dosage of Phe and Ur applied to the grapevines gave rise to a higher threonine and lysine content in the resultant musts. Therefore, it is probable that acetoin formation is related to all the aforementioned factors rather than to the amino acids individually.

Phenylalanine and urea have been applied to grapevines as fertilisers, with different results in the volatile composition of grapes and wines. Ancín-Azpilicueta et al. (2013) studied the effect of fertilisation of Tempranillo grapevines with two doses of urea on wine volatile compounds. These authors reported that the total content of alcohols in the wines decreased with the application of urea to the vineyard. However, the concentrations of ethyl hexanoate, ethyl octanoate and ethyl decanoate were highest in the wines originating from vines that received the highest dosage of urea. In this work, fatty acids presented different individual responses to the urea foliar applications. Garde-Cerdán et al. (2015a) showed that phenylalanine treatment applied to Tempranillo grapevines increased the presence of benzenoids and decreased C6 compounds in the grapes. However, the same trend was not observed in our work, and both doses of urea and phenylalanine applied to the grapevines barely affected the wine volatile concentration, as was observed and discussed previously in the first study season.

\section{Treatment classification}

Principal component analysis (PCA) was performed on the concentrations of higher alcohols, fatty acids, acetate esters, ethyl esters and other volatile compounds in wines from untreated grapevines (control) and grapevines treated (independent variables) with several nitrogen sources such as proline (Pro), phenylalanine (Phe), urea (Ur), commercial products without amino acids (Cp), and commercial products with amino acids (Cpaas), applied during the first study year, and the results are shown in Fig. 1a. The PCA of the two different doses of phenylalanine (Phe1: low, and Phe2: high) and urea (Ur1: low, and Ur2: high) applied during the second study year are shown in Fig. 1b. In the case of several nitrogen sources, principal component 1 (PC1) explained $44.0 \%$ of the variance and PC2 explained $24.5 \%$ of the variance, representing $68.5 \%$ of all variance (Fig. 1a). Both components made it possible to separate the treatments. PC1 was strongly correlated with isoamyl alcohols, isobutanol, 2-phenylethanol, benzyl alcohol, methionol, hexyl acetate, 2-phenylethyl acetate, acetaldehyde, diacetyl and $\gamma$-butyrolactone; while PC2 was strongly correlated with octanoid acid, ethyl 3-hydroxybutyrate, ethyl hexanoate and ethyl octanoate. The control wines were correlated with a high content of several volatile compounds, such as methionol, hexyl acetate, 2-phenylethyl acetate, diacetyl and $\gamma$-butyrolactone (Fig. 1a). The Pro and Cp wines were correlated with ethyl octanoate. The Ur wines were correlated with isoamyl acetate, while the Phe wines were 


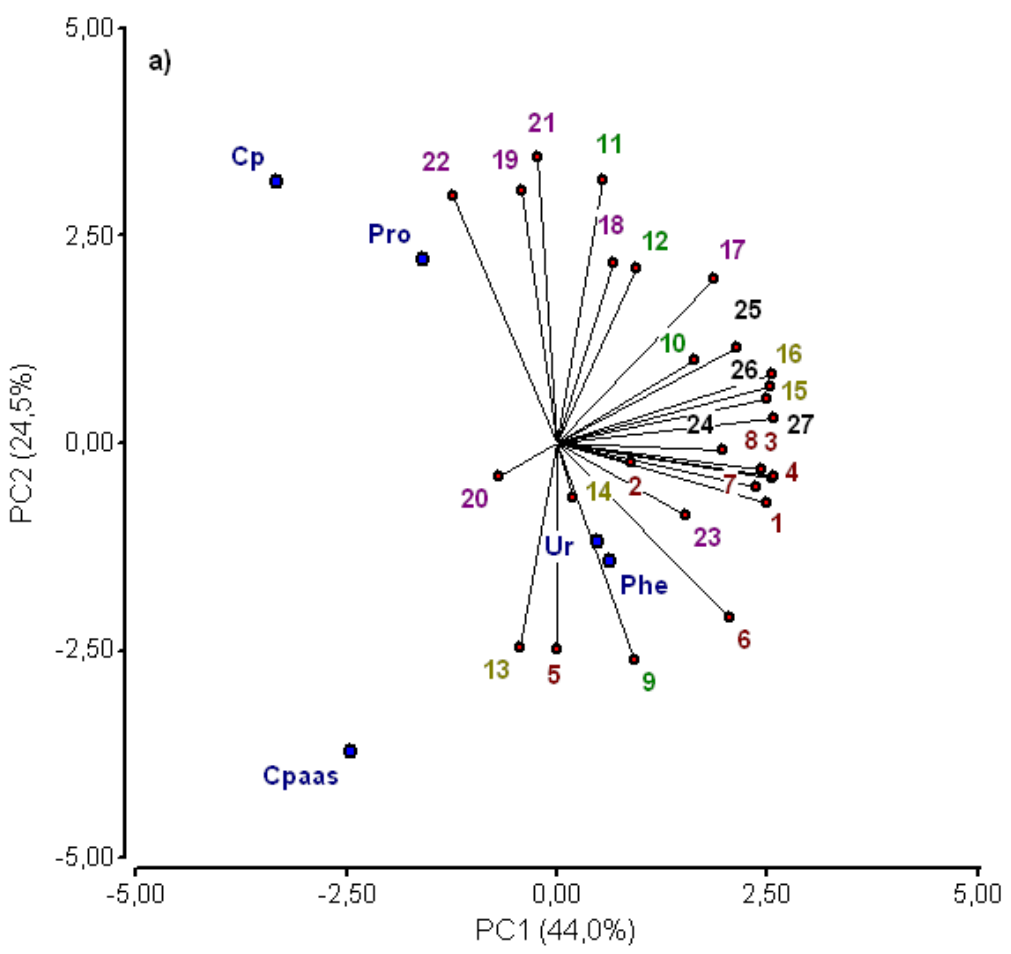

FIGURE 1a

PCA of volatile compound concentration $(\mathrm{mg} / \mathrm{L})$ in the different Tempranillo samples: control and treated with proline (Pro), phenylalanine (Phe), urea (Ur), commercial product without amino acids (Cp), and commercial product with amino acids (Cpaas). The numbers correspond to the volatile compounds in the order that they appear in Tables 1 and 2. Numbers in orange correspond to higher alcohols, in green to volatile fatty acids, in yellow to acetate esters, in purple to ethyl esters, and in black to other volatile compounds.

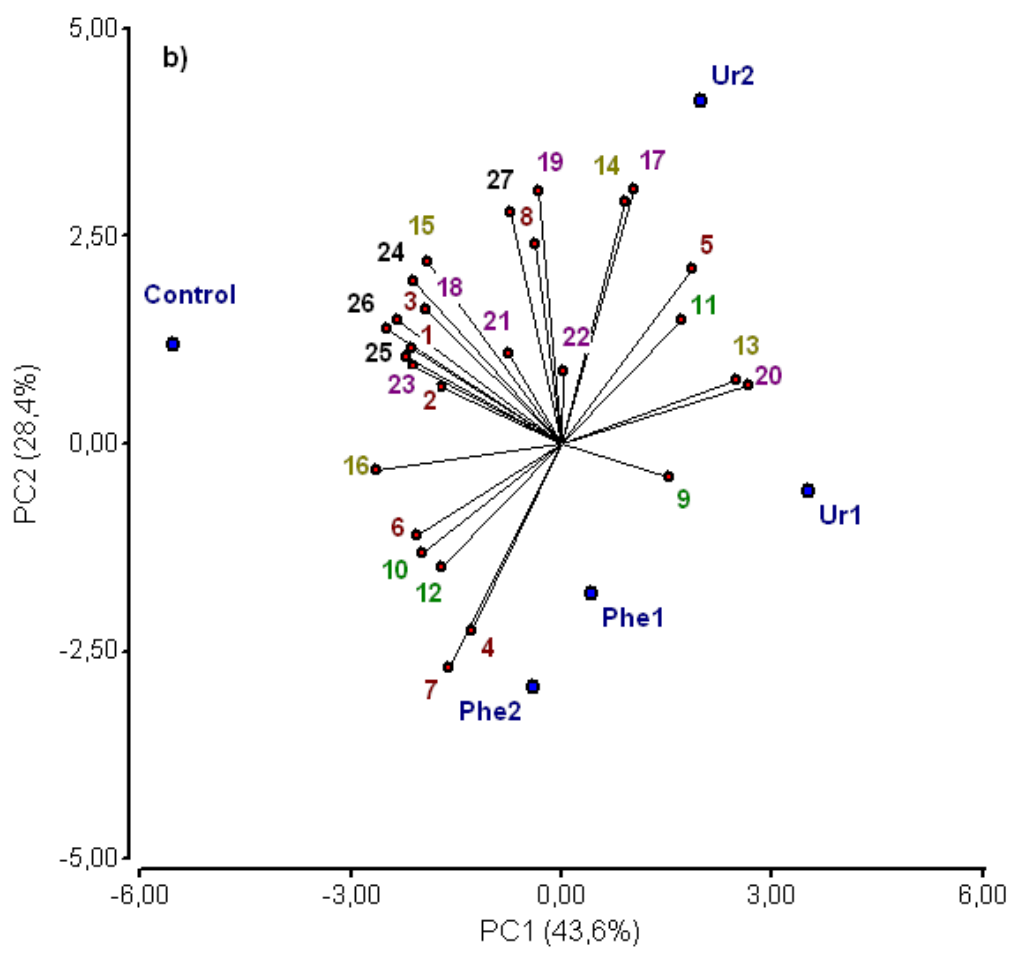

FIGURE $1 \mathrm{~b}$

PCA of volatile compound concentration $(\mathrm{mg} / \mathrm{L})$ in the different Tempranillo samples: control, and treated with two doses of phenylalanine (Phe1 and Phe2) and two doses of urea (Ur1 and Ur2). The numbers correspond to the volatile compounds in the order that they appear in Tables 1 and 2. Numbers in orange correspond to higher alcohols, in green to volatile fatty acids, in yellow to acetate esters, in purple to ethyl esters, and in black to other volatile compounds. 
correlated with n-butanol and isobutyric acid. Cpaas wines were correlated with n-hexanol and ethyl acetate.

For the grapevines treated with the two doses of Phe and Ur, principal component 1 (PC1) explained $43.6 \%$ of the variance and PC2 explained $28.4 \%$ of the variance, representing $72.0 \%$ of all variance (Fig. 1b). Both components made it possible to separate the wines from the different treatments. PC1 was strongly correlated with isobutanol, ethyl acetate, 2-phenylethyl acetate, ethyl butyrate, diacetyl and acetoine; while PC2 was strongly correlated with benzyl alcohol, hexyl acetate, ethyl propionate and ethyl 3-hydroxybutyrate. The control wines were correlated with a high content of several volatile compounds, such as isoamyl alcohols, n-propanol, isobutanol, ethyl isobutyrate, diethyl succinate, diacetyl and acetoine (Fig. 1b). The Phel and Phe2 wines were correlated with 2-phenylethanol and benzyl alcohol. The Ur1 wines were correlated with isobutyric acid, while the Ur2 wines were correlated with isoamyl acetate and ethyl propionate.

Higher alcohols and esters in wines are derived from the metabolism of sugars and amino acids, while other esters are derived from grape glycosides (Swiegers et al., 2005). These volatile compounds confer the generic or fermentation bouquet in wines, usually contributing to floral and fruity aroma (Noguerol-Pato et al., 2014). Nitrogen composition and fertilisation can result in an increase in wine ester concentration. However, the effects on the content of higher alcohols shows contradictory effects (Bell \& Henschke, 2005; Gutiérrez-Gamboa et al., 2018), which probably depend on the grape nitrogenous composition. Based on the nitrogen composition of the grapevines under study reported in others works (Garde-Cerdán et al., 2014a, 2018), the concentration of total amino acids found in the musts was largely above $140 \mathrm{mg} \mathrm{N} / \mathrm{L}$. This concentration has been reported as necessary for carrying out a correct alcoholic fermentation to avoid stuck and sluggish fermentations (Bisson \& Butzke, 2000; Bell \& Henschke, 2005). Several authors have found a direct relationship between must nitrogen concentration and the concentration of ethyl esters in wines, except at high nitrogen concentrations (Hernández-Orte et al., 2002; Bell \& Henschke, 2005; Martínez-Gil et al., 2012; GutiérrezGamboa et al., 2018). Likewise, the nitrogen demand of yeast might also affect higher alcohols and the production of ethyl and acetate esters. Torrea et al. (2003) reported that a strain with a high nitrogen demand compared to one with a lower demand produced higher total esters and lower total higher alcohols, even though some volatiles showed strong variation. The commercial Saccharomyces cerevisiae strain Uvaferm VRB has intermediate needs for assimilable nitrogen. It therefore is possible that nitrogen fertilisation did not affect wine volatile composition because the grapevines had low nitrogen needs. The reason for this is that the yeast starter culture was consistent for all treatments.

\section{CONCLUSIONS}

Foliar nitrogen applications of several nitrogen sources to Tempranillo grapevines studied during the first season had a limited effect on wine volatile composition. Furthermore, urea and phenylalanine foliar applications to Tempranillo grapevines at two dosages during the second vintage barely affected wine volatile composition. These results have viticultural and oenological interest, as the nitrogen treatments applied during this study allow for the improvement of wine amino acid and phenolic composition, without affecting their volatile composition.

\section{LITERATURE CITED}

Ancín-Azpilicueta, C., Nieto-Rojo, R. \& Gómez-Cordón, J., 2013. Effect of foliar urea fertilisation on volatile compounds in Tempranillo wine. J. Sci. Food Agric. 93, 1485-1491.

Barbosa, C., Falco, V., Mendes-Faia, A. \& Mendes-Ferreira, A., 2009. Nitrogen addition influences formation of aroma compounds, volatile acidity and ethanol in nitrogen deficient media fermented by Saccharomyces cerevisiae wine strains. J. Biosci. Bioeng. 108, 99-104.

Bell, S.J. \& Henschke, P.A., 2005. Implications of nitrogen nutrition for grapes, fermentation and wine. Aust. J. Grape Wine Res. 11, 242-295.

Bisson, L.F. \& Butzke, C.E., 2000. Diagnosis and rectification of stuck and sluggish fermentations. Am. J. Enol. Vitic. 51, 168-177.

Cai, J., Zhu, B.-Q., Wang, Y.-H., Lu, L., Lan, Y.-B., Reeves, M.J. \& Duan, C.-Q., 2014. Influence of pre-fermentation cold maceration treatment on aroma compounds of Cabernet Sauvignon wines fermented in different industrial scale fermenters. Food Chem. 154, 217-229.

Carrau, F.M., Medina, K., Farina, L., Boido, E., Henschke, P.A., \& Dellacassa, E., 2008. Production of fermentation aroma compounds by Saccharomyces cerevisiae wine yeasts: Effects of yeast assimilable nitrogen on two model strains. FEMS Yeast Res. 8, 1196-1207.

Christensen, L.P. \& Peacock, W.L., 2000. Raisin production manual. In: Christensen, L.P. (ed.). Mineral nutrition and fertilization. Oakland, California : University of California, Agricultural and Natural Resources Publication. pp. $102-114$.

Corison, C.A., Ough, C.S., Berg, H.W. \& Nelson, K.E., 1979. Must acetic acid and ethyl acetate as mold and rot indicators in grapes. Am. J. Enol. Viti. 30, 130-134.

Culleré, L., Escudero, A., Cacho, J. \& Ferreira, V. 2004. Gas chromatography-olfactometry and chemical quantitative study of the aroma of six premium quality Spanish aged red wines. J. Agric. Food Chem. 52(6), 1653-1660.

Dennis, E.G., Keyzers, R.A., Kalua, C.M., Maffei, S.M., Nicholson, E.L. \& Boss, P.K., (2012). Grape contribution to wine aroma: Production of hexyl acetate, octyl acetate, and benzyl acetate during yeast fermentation is dependent upon precursors in the must. J. Agric. Food Chem. 60, 2638-2646.

Ferreira, V., López, R., Cacho. JF. 2000. Quantitative determination of the odorants of young red wines from different grape varieties. J. Sci Food Agric. $80,1659-1667$.

Garde-Cerdán, T. \& Ancín-Azpilicueta, C., 2008. Effect of the addition of different quantities of amino acids to nitrogen-deficient must on the formation of esters, alcohols, and acids during wine alcoholic fermentation. LWTFood Sci. Technol. 41, 501-510.

Garde-Cerdán, T., Jarauta, I., Salinas, M.R., \& Ancín-Azpilicueta, C., 2008. Comparative study of the volatile composition in wines obtained from traditional vinification and from the Ganimede method. J. Sci. Food Agric. 88, 1777-1785.

Garde-Cerdán, T., López, R., Portu, J., González-Arenzana, L., López-Alfaro, I. \& Santamaría, P., 2014a. Study of the effects of proline, phenylalanine, and urea foliar application to Tempranillo vineyards on grape amino acid content. Comparison with commercial nitrogen fertilisers. Food Chem. 163, 136-141. 
Garde-Cerdán, T., López, R., Garijo, P., González-Arenzana, L., Gutiérrez, A. R., López-Alfaro, I. \& Santamaría, P., 2014b. Application of colloidal silver versus sulfur dioxide during vinification and storage of Tempranillo red wines. Aust. J. Grape Wine Res. 20, 51-61.

Garde-Cerdán, T., Santamaría, P., Rubio-Bretón, P., González-Arenzana, L., López-Alfaro, I. \& López, R., 2015a. Foliar application of proline, phenylalanine, and urea to Tempranillo vines: Effect on grape volatile composition and comparison with the use of commercial nitrogen fertilizers. LWT-Food Sci. Technol. 60, 684-689.

Garde-Cerdán, T., Portu, J., López, J. \& Santamaría, P., 2015b. Impact of foliar applications of proline, phenylalanine, urea, and commercial nitrogen fertilizers on the stilbene concentration of Tempranillo musts and wines. Am. J. Enol. Vitic. 66, 542-546.

Garde-Cerdán, T., Gutiérrez-Gamboa, G., Pérez-Álvarez, E.P. \& López, R., 2018. Influence of foliar application of phenylalanine and urea at two doses to vineyards on grape volatile composition and amino acids content. Vitis. Submitted.

Guth, H., 1997. Quantitation and sensory studies of character impact odorants of different white wine varieties. J. Agric. Food Chem. 45, 3027-3032.

Gutiérrez-Gamboa, G., Portu, J., Santamaría, P., López, R. \& Garde-Cerdán, T., 2017a. Effects on grape amino acid concentration through foliar application of three different elicitors. Food Res. Int. 99, 688-692.

Gutiérrez-Gamboa, G., Portu, J., López, R., Santamaría, P. \& Garde-Cerdán, T., 2017b. Elicitor and nitrogen applications to Garnacha, Graciano, and Tempranillo vines: Effect on grape amino acid composition. J. Sci. Food Agric. 98(6), 2341-2349.

Gutiérrez-Gamboa, G., Garde-Cerdán, T., Portu, J., Moreno-Simunovic, Y \& Martínez-Gil, A.M., 2017c. Foliar nitrogen application in Cabernet Sauvignon vines: Effects on wine flavonoid and amino acid content. Food Res. Int. 96, 46-53.

Gutiérrez-Gamboa, G., Garde-Cerdán, T., Moreno-Simunovic, Y. \& Martínez-Gil, A.M., 2018. Improvement of wine volatile composition through foliar nitrogen applications to 'Cabernet Sauvignon' grapevines in a warm climate. Chil. J. Agr. Res. In press.

Hannam, K., Nielsen, G.H., Nielsen, D., Midwood, A.J., Millard, P., Zhang, Z., Thornton, B. \& Steinke, D., 2016. Amino acid composition of grape ( $\mathrm{Vi}$ tis vinifera L.) juice in response to applications of urea to the soil or foliage. Am. J. Enol. Vitic. 67, 47-55

Hernández-Orte, P., Cacho, J. \& Ferreira, V., 2002. Relationship between varietal amino acid profile of grapes and wine aromatic composition. Experiments with model solutions and chemometric study. J. Agric. Food Chem. 50, 2891-2899.

Lacroux, F., Tregoat, O., Van Leeuwen, C., Pons, A., Tominaga, T., Lavigne-Cruège, V. \& Dubourdieu, D., 2008. Effect of foliar nitrogen and sulphur application on aromatic expression of Vitis vinifera L. cv. Sauvignon blanc. J. Int. Sci. Vigne Vin, 42(3), 125-132.

Lasa, B., Menendez, S., Sagastizabal, K., Cervantes, M.E.C., Irigoyen, I., Muro, J., Aparicio-Tejo, P.M. \& Ariz, I., 2012. Foliar application of urea to "Sauvignon Blanc" and "Merlot" vines: Doses and time of application. Plant Growth Regul. 67, 73-81.

Martineau, B., Terry, E. \& Henick-King, T., 1995. Effect of wine type on the detection threshold for diacetyl. Food Res. Int. 28, 139-143.
Martínez-Gil, A.M., Garde-Cerdán, T., Lorenzo, C., Lara, J.F., Pardo, F. \& Salinas, M.R., 2012. Volatile compounds formation in alcoholic fermentation from grapes collected at 2 maturation stages: Influence of nitrogen compounds and grape variety. J. Food Sci. 77, 71-79.

Noguerol-Pato, R., Sieiro-Sampedro, T., González-Barreiro, C., CanchoGrande, B. \& Simal-Gándara, J., 2014. Effect on the aroma profile of Graciano and Tempranillo red wines of the application of two antifungal treatments onto vines. Molecules 19, 12173-12193.

Pineau, B., Barbe, J.C., Van Leeuwen, C. \& Dubourdieu, D., 2009. Examples of perceptive interactions involved in specific "red-" and "black-berry" aromas in red wines. J. Agric. Food Chem. 57, 3702-3708.

Romano, P. \& Suzzi, G., 1996. Origin and production of acetoin during wine yeast fermentation. Appl. Environ. Microbiol. 62, 309-315.

Sampaio, T.L., Kennedy, J.A. \& Vasconcelos, M.C., 2007. Use of microscale fermentations in grape and wine research. Am. J. Enol. Vitic. 58, 534-539.

Smith, M.E., Bekker, M.Z., Smith, P.A. \& Wilkes, E.N., 2015. Sources of volatile sulfur compounds in wine. Aust. J. Grape Wine Res. 21, 705-712.

Soubeyrand, E., Basteau, C., Hilbert, G., Van Leeuwen, C., Delrot, S. \& Gomès, E., 2014. Nitrogen supply affects anthocyanin biosynthetic and regulatory genes in grapevine cv. Cabernet-Sauvignon berries. Phytochem. $103,38-49$

Swiegers, J.H., Bartowsky, E.J., Henschke, P.A. \& Pretorius, I.S., 2005. Yeast and bacterial modulation of wine aroma and flavour. Aust. J. Grape Wine Res. 11, 139-173.

Torrea, D., Fraile, P., Garde-Cerdán, T. \& Ancín-Azpilicueta, C., 2003 Production of volatile compounds in the fermentation of chardonnay must inoculated with two strains of Saccharomyces cerevisiae with different nitrogen demands. Food Control 14, 565-571.

Ugliano, M., Fedrizzi, B., Siebert, T., Travis, B., Magno, F., Versini, G. \& Henschke, P.A., 2009. Effect of nitrogen supplementation and Saccharomyces species on hydrogen sulfide and other volatile sulfur compounds in Shiraz fermentation and wine. J. Agric. Food Chem. 57, 4948-4955

Verdenal, T., Spangenberg, J.E., Zufferey, V., Lorenzini, F., Spring, J.L. \& Virent, O., 2015. Effect of fertilisation timing on the partitioning of foliar applied nitrogen in Vitis vinifera cv. Chasselas: A ${ }^{15} \mathrm{~N}$ labelling approach. Aust. J. Grape Wine Res. 21, 110-117.

Vilanova, M., Ugliano, M., Varela, C., Siebert, T., Pretorius, I.S. \& Henschke, P.A., 2007. Assimilable nitrogen utilisation and production of volatile and non-volatile compounds in chemically defined medium by Saccharomyces cerevisiae wine yeasts. Appl. Microbiol. Biotechnol. 77, 145-157.

Vilanova, M., Masa, A. \& Tardaguila, J., 2009. Evaluation of the aromatic variability of Spanish grape by quantitative descriptive analysis. Euphytica $165,383-389$.

Vilanova, M., Genisheva, Z., Graña, M. \& Oliveira, J.M., 2013. Determination of odorants in varietal wines from international grape cultivars (Vitis vinifera) grown in NW Spain. S. Afr. J. Enol. Vitic. 34, 212 222 .

Zea, L., Serratosa, M.P., Mérida, J. \& Moyano, L., 2015. Acetaldehyde as key compound for the authenticity of sherry wines: A study covering 5 decades. Comp. Rev. Food Sci. Food Safety 14, 681-693. 